\title{
Ultrasound biomicroscopy of eyes undergoing deep sclerectomy with collagen implant
}

Hôpital Ophtalmique Jules Gonin, Department of Ophthalmology, University of Lausanne, Lausanne, Switzerland A G-Y Chiou A Mermoud S-E A Hédiguer C C Schnyder R Faggioni

Correspondence to: André Mermoud, $\mathrm{MD}$, Hôpital Ophtalmique Jules Gonin, Avenue de France, 15, 1004 Lausanne, Switzerland.

Auguste G-Y Chiou, André Mermoud, Serge-Emmanuel A Hédiguer, Corinne C Schnyder, Ruggero Faggioni

\begin{abstract}
Aims-To assess the intraocular pressure (IOP) lowering mechanism of deep sclerectomy with collagen implant (DSCI), a non-penetrating glaucoma surgery.

Methods-Nine eyes of nine patients with medically uncontrolled open angle glaucoma underwent DSCI. Ultrasound biomicroscopy (UBM) of the sclerectomy site was performed 1 month after surgery. The following factors were assessed: length and height of collagen implant, and thickness of the residual trabeculocorneal membrane.
\end{abstract}

Results-Postoperative IOP decreased significantly in all nine eyes from a preoperative mean value of 25.8 (SD 4.8 ) $\mathrm{mm}$ $\mathrm{Hg}$ to a postoperative (1 month) mean value of $11.3(6.3) \mathrm{mm} \mathrm{Hg}(p=0.001)$. In all nine eyes, UBM at 1 month after surgery showed a subconjunctival filtration through the thin trabeculocorneal membrane and through the scleral flap around the collagen implant. In four cases, a hypoechoic area in the suprachoroidal space was observed and might represent ciliary body detachment or be due to suprachoroidal drainage of aqueous humour through the thin deep scleral wall. At 1 month after surgery the mean trabeculocorneal membrane thickness was $110.1(16.8) \mu \mathrm{m}$, and the mean length and height of the collagen implant were 2.3 $(0.1) \mathrm{mm}$ and $1.1(0.1) \mathrm{mm}$ respectively. Conclusion-DSCI lowered 1OP by allowing aqueous filtration through $a$ thin trabeculocorneal membrane to the subconjunctival space and, eventually, to the suprachoroidal space.

(Br F Ophthalmol 1996;80:541-544)

Deep sclerectomy with collagen implant (DSCI), a non-penetrating glaucoma surgery, was first described by Kozlov et al in $1989 .{ }^{1}$ In this technique, a deep sclerectomy with thinning of the adjacent trabeculum and limbal cornea is performed. Although still under investigation, this procedure seems to be promising as it lowers the intraocular pressure (IOP) without perforation of the anterior chamber, thus reducing the occurrence of postoperative complications.

High frequency ultrasound biomicroscopy (UBM) allows detailed anatomical assessment of the anterior eye segment. ${ }^{2}$ In order to deter- mine the IOP lowering mechanism of this new technique, we performed a UBM scan of the sclerectomy site in nine eyes, 1 month after DSCI.

\section{Materials and methods}

PATIENTS

Nine eyes of nine consecutive patients underwent successful DSCI. There were four men and five women, with a mean age of 70.3 (SD 12.3) years (range 49 to 80 years). Six eyes had primary open angle glaucoma, two eyes had capsular glaucoma, and one eye had pseudophakic glaucoma (Table 1). Complete eye examination was performed before surgery and 1 day, 1 week, and 1 month postoperatively. UBM of the sclerectomy site was performed 1 month postoperatively.

\section{SURGERY}

All patients underwent DSCI under retrobulbar anaesthesia with bupivacaine $0.75 \%$ -lignocaine $4 \%$ and hyaluronidase 50 IU. The same surgeon (AM) performed all surgeries in the following way. After opening the conjunctiva in the upper fornix, a scleral flap measuring $5 \mathrm{~mm}$ by $5 \mathrm{~mm}$ was created using a diamond knife. The scleral flap had a thickness of approximately $40 \%$ scleral thickness. A deep sclerectomy was then performed, leaving a thin scleral layer over the choroid and ciliary body. The Schlemm's canal was 'unroofed', and the corneal stroma was removed anteriorly down to the Descemet's membrane. At that stage of the procedure, aqueous humour was percolating through the thin trabecular corneal membrane. The collagen implant (Staar Surgica AG, Nidau, Switzerland) was then secured to the deep sclera by placing a $10 / 0$ nylon suture at the midpoint of the triangular bed. The rectangular scleral flap was then closed and sutured with two 10/0 nylon sutures. The Tenon's capsule and the conjunctiva were closed with separate $8 / 0$ silk sutures. Neither 5-fluorouracil nor mitomycin $\mathrm{C}$ had been used. Postoperatively the patients were treated with topical application of dexamethasone, neomycin, and polymyxin B drops (Maxitrol) four times daily for 1 month, followed by fluorometholone drops (Efemoline) three times daily for 5 months.

\section{COLLAGEN IMPLANT}

The device is manufactured from microporous crosslinked collagen harvested from porcine sclera. In its dry state, the collagen device measures approximately $2.5 \mathrm{~mm} \times 1.0 \mathrm{~mm} \times 0.5$ 
Table 1 Patient data

\begin{tabular}{llll}
\hline Patient No & Sex & Age (years) & $\begin{array}{l}\text { Type of } \\
\text { glaucoma }\end{array}$ \\
\hline 1 & Male & 75 & POAG \\
2 & Male & 71 & $\begin{array}{l}\text { Capsular } \\
\text { glaucoma } \\
\text { Pseudophakic } \\
\text { glaucoma }\end{array}$ \\
3 & Male & 80 & POAG \\
4 & Male & 49 & POAG \\
5 & Female & 80 & POAG \\
6 & Female & 79 & Capsular \\
7 & Female & 68 & POAG \\
8 & Female & 80 & POAG \\
9 & Female & 51 & \\
\hline
\end{tabular}

POAG = primary open angle glaucoma

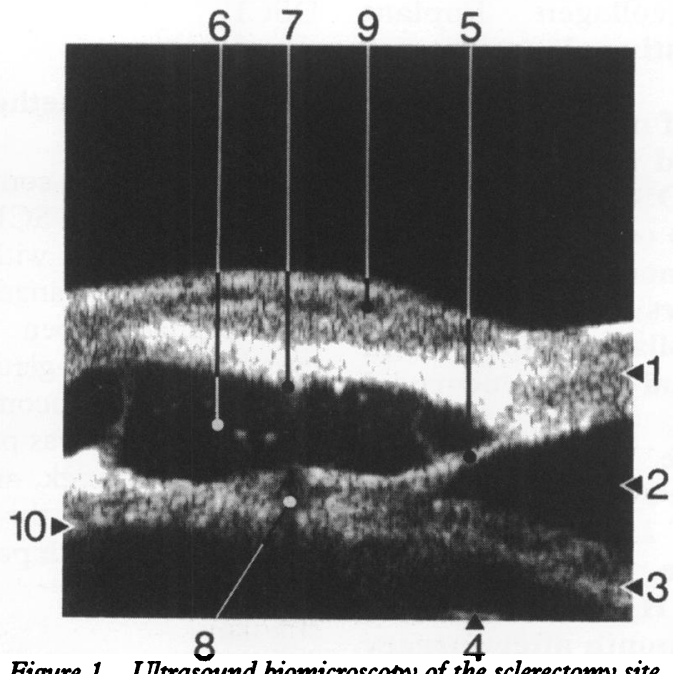

Figure 1 Ultrasound biomicroscopy of the sclerectomy site 1 month after deep sclerectomy with collagen implant. The collagen implant maintains the pathway under the scleral flap. The anterior chamber is not perforated. (1) Cornea, (2) anterior chamber, (3) iris, (4) lens, (5) trabeculocorneal membrane, (6) collagen implant, (7) implant narrowing caused by the scleral suture, (8) acoustic shadowing caused by the scleral suture, (9) conjunctival bleb, (10) choroid.

mm. After placement in the subscleral space and exposure to ocular fluids, the device is intended to swell approximately two to three times its dry size as it absorbs fluid. It prevents reattachment of the scleral flap to the deeper sclera.

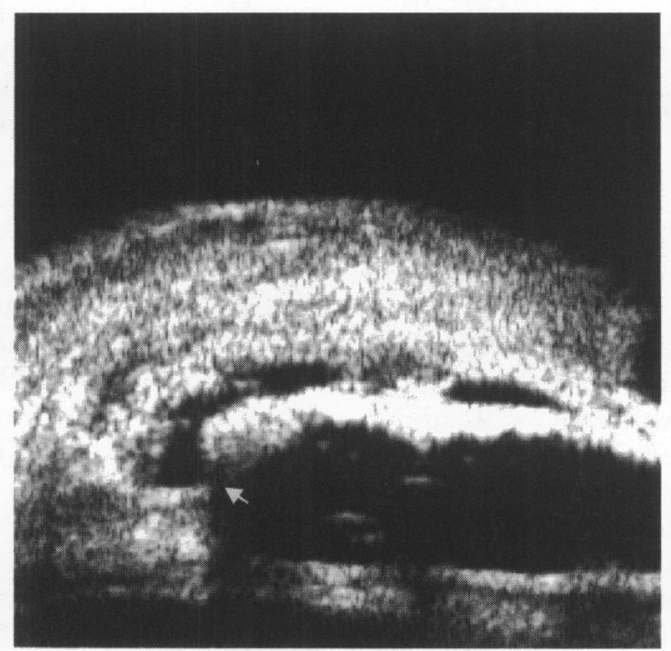

Figure 2 Drainage pathway (arrow) of aqueous through the scleral flap.

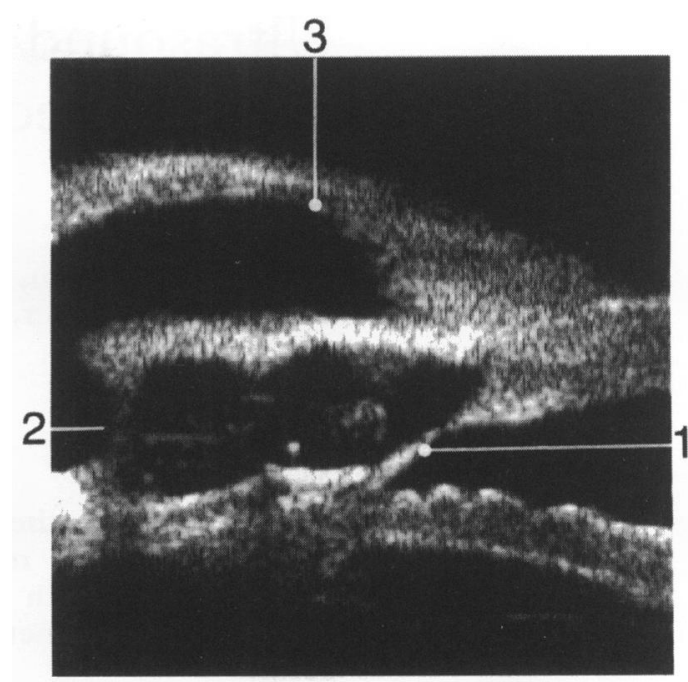

Figure 3 This picture shows the non-perforation of the eye and the drainage of aqueous through the scleral flap to the conjunctival bleb. (1) Trabeculocorneal membrane, (2) drainage of aqueous through the scleral flap, (3) conjunctival bleb.

ULTRASOUND BIOMICROSCOPY

The Humphrey UBM 840 system (Humphrey Instruments Inc, San Leandro, CA, USA) was used to obtain high frequency ( $50 \mathrm{MHz}$ ) ultrasonic scan images. As the interobserver reliability varies considerably and is affected by subjective interpretation of visualised anatomical landmarks, ${ }^{3}$ all the examinations were performed by one single observer (AM). The following factors were assessed: length and height of the collagen implant (measured at its largest point), and thickness of the residual trabeculocorneal membrane (measured at its thinnest point).

\section{STATISTICS}

Data were compared using the Student's $t$ test. A difference was considered significant when $p$ was lower than 0.05 .

\section{Results}

Detailed results for each patient are reported in Table 2. Postoperative IOP decreased significantly in all nine eyes from a preoperative mean value of 25.8 (4.8) $\mathrm{mm} \mathrm{Hg}$ to a postoperative ( 1 month) mean value of $11.3(6.3) \mathrm{mm}$ $\mathrm{Hg}(\mathrm{p}=0.001)$. The high postoperative IOP $(25$ $\mathrm{mm} \mathrm{Hg}$ at 1 month) of patient 2 was due to a corticosteroid response. After discontinuation of the steroid treatment, IOP normalised to 12 $\mathrm{mm} \mathrm{Hg}$ and $13 \mathrm{~mm} \mathrm{Hg}$ at 2 and 3 months postoperatively respectively. No other complication was noted.

In all nine eyes, UBM showed a subconjunctival filtration through the thin trabeculocorneal membrane and through the scleral flap around the collagen implant (Figs 1, 2, and 3).

In four cases, a suprachoroidal hypoechoic area was clearly observed under the sclerectomy site as represented in Figure 4. After exclusion of the eye with a corticosteroid response, the four eyes with a hypoechoic area had a lower postoperative IOP compared with the four other eyes (mean IOP $6.3(1.7) \mathrm{mm}$ $\mathrm{Hg}$ versus 13 (1.2) $\mathrm{mm} \mathrm{Hg}, \mathrm{p}=0.001$ ). 
Table 2 Characteristics of eyes after deep sclerectomy with collagen implant

\begin{tabular}{|c|c|c|c|c|c|c|c|}
\hline Patient No & $\begin{array}{l}\text { Preoperative } \\
I O P(\mathrm{~mm} \mathrm{Hg})\end{array}$ & $\begin{array}{l}\text { IOP at } \\
1 \text { month } \\
(\mathrm{mm} \mathrm{Hg})\end{array}$ & $\begin{array}{l}\text { Trabeculocorneal } \\
\text { membrane } \\
\text { thickness }(\mu m)\end{array}$ & $\begin{array}{l}\text { Collagen } \\
\text { implant length } \\
\text { (mm) }\end{array}$ & $\begin{array}{l}\text { Collagen } \\
\text { implant height } \\
\text { (mm) }\end{array}$ & $\begin{array}{l}\text { Subconjunctival } \\
\text { filtration }\end{array}$ & $\begin{array}{l}\text { Suprachoroidal } \\
\text { hypoechoic area }\end{array}$ \\
\hline 1 & 27 & 14 & 99 & 2.2 & 1.1 & + & - \\
\hline 2 & 26 & 25 & 109 & 2.2 & 1 & + & - \\
\hline 3 & 25 & 12 & 129 & 2.3 & 1.1 & + & - \\
\hline 4 & 28 & 7 & 116 & 2.3 & 1.2 & + & + \\
\hline 5 & 18 & 14 & 104 & 2.3 & 1.3 & + & - \\
\hline 6 & 25 & 6 & 95 & 2.4 & 1.1 & + & + \\
\hline 7 & 25 & 12 & 121 & 2.4 & 1.2 & + & - \\
\hline 8 & 22 & 8 & 135 & 2.3 & 1.1 & + & + \\
\hline 9 & 36 & 4 & 83 & 2.6 & 1.1 & + & + \\
\hline
\end{tabular}

The mean trabeculocorneal membrane thickness was 110.1 (16.8) $\mu \mathrm{m}$ ( range 83-135 $\mu \mathrm{m})$. The mean length and height of the collagen implant were $2.3(0.1) \mathrm{mm}$ ( range $2.2-2.6 \mathrm{~mm}$ ) and $1.1(0.1) \mathrm{mm}$ (range 1-1.3 $\mathrm{mm}$ ) respectively.

\section{Discussion}

The main advantage of DSCI over standard trabeculectomy is the non-perforation of the anterior chamber, allowing a progressive decrease of IOP and, thus, lowering the occurrence of complications such as hyphaema, flat anterior chamber, and anterior chamber inflammation. The collagen implant is slowly dissolved within 3 to 6 months and replaced by new autologous scleral tissue, leaving a tunnel in the sclera. In order to study the IOP lowering mechanism of DSCI, the use of a high resolution device, such as the ultrasound biomicroscope, was necessary.

In eight eyes, IOP decreased significantly 1 month after surgery. One eye presented a high postoperative IOP because of a corticosteroid response. This complication may occur after a successful filtering surgery, as described previously after trabeculectomy ${ }^{4}$ or implantation of a Molteno tube. ${ }^{5}$

UBM imaging showed that DSCI lowered IOP by allowing aqueous filtration through a thin remaining trabeculocorneal membrane to

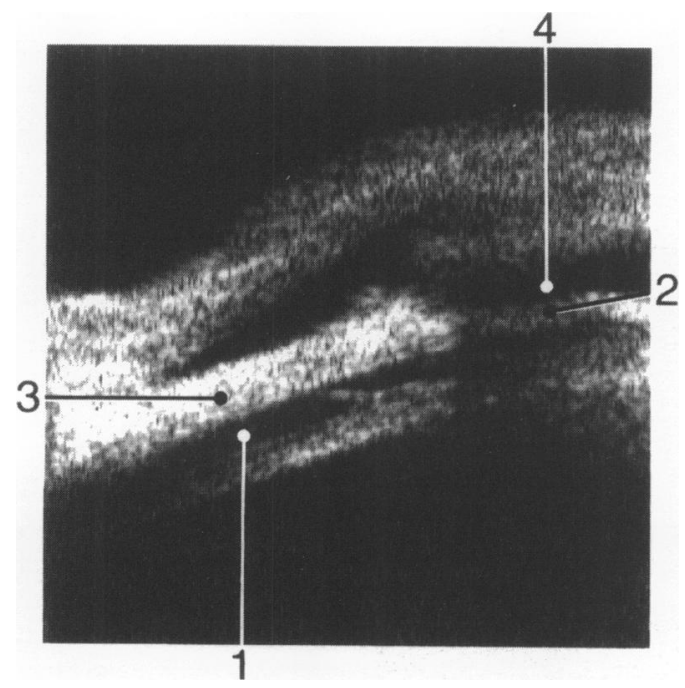

Figure 4 Suprachoroidal hypoechoic area posterior to the sclerectomy site. This looks like a ciliary body detachment or may represent a choroidal filtration of aqueous through the thin deep sclera. (1) Suprachoroidal hypoechoic area, (2) deep sclera, (3) sclera posterior to the sclerectomy site, (4) aqueous under the scleral flap. an area under the scleral flap, which was maintained open by the collagen implant. The aqueous humour then reached the subconjunctival space. We also observed a hypoechoic area in the suprachoroidal space in four cases (44\%). This aspect might represent a ciliary body detachment or a suprachoroidal drainage of aqueous humour. The aqueous humour under the scleral flap was separated from the choroid by a thin scleral wall and might filter to the suprachoroidal space, causing detachment of the ciliary body. This phenomenon was associated with lower IOP, either as a result of ciliary body detachment or because of choroidal resorption of aqueous humour. A combined (subconjunctival and suprachoroidal) outflow pathway might offer a better IOP reduction compared with standard trabeculectomy. However, further studies are needed to evaluate this hypothesis.

UBM was performed 1 month after surgery. Therefore, the thickness of the trabeculocorneal membrane, and the length and height of the collagen implant represented single measurements. These factors may vary in time, and further studies are needed to assess the structural evolution of eyes undergoing DSCI. Measurements using electronic calipers on a screen may be accurate provided the measurements are made in axis with the beam or perpendicular to the beam. In this study the measurements of the height and width of the implant could be considered to be accurate. However, the residual trabeculocorneal membrane is angled to the transducer and of a size approaching the minimum resolvable measurement of the instrument. Its value should be considered as an approximation.

DSCI represents a delicate microsurgical technique that requires surgical skills. The main difficulty is to perform the dissection of the deep sclera and to avoid a perforation of the remaining trabeculocorneal membrane. As aqueous humour has to reach the iridocorneal angle to filter through the trabeculocorneal membrane and because in this procedure no iridectomy is performed, DSCI is not recommended in patients with angle closure glaucoma.

UBM was a useful method of assessing the anatomical results of eyes undergoing DSCI and allowed us to understand the aqueous humour outflow mechanism of this new, non-perforating filtration surgery 1 month after surgery. Further studies are needed to assess the long term functional and anatomical results of DSCI. 
The authors have no proprietary interest in the development of or the marketing of any commercial device named in the study.

1 Kozlov VI, Bagrov SN, Anisimova SY, Osipov AV, Mogilevtsev VV. Non penetrating deep sclerectomy with collagen. IRTC Eye Microsurgery. Moscow: RSFSR Ministry of PubIRTC Eye Microsurgery.
lic Health, 1989:44-6.

2 Pavlin CJ, Harasiewicz K, Eng P, Sherar MD, Foster FS. Clinical use of ultrasound biomicroscopy. Ophthalmology 1991;98:287-95.
3 Tello C, Liebmann J, Potash SD, Cohen H, Ritch R. Measurement of ultrasound biomicroscopy images: intraobserver and interobserver reliability. Invest Ophthalmol Vis Sci1 994:35:3549-52.

4 Wilensky JT, Snyder D, Gieser D. Steroid-induced ocular hypertension in patients with filtering blebs. Ophthalmology 1980;87:240-4

5 Mermoud A, Salmon J. Corticosteroid induced ocular hypertension in draining Molteno single-plate implants. $\mathcal{F}$ Glaucoma 1993;2:32-6. 